\title{
Producción e impacto del biodiesel: una revisión
}

\section{Production and impact of biodiesel: a review}

\author{
Edilberto Antonio Llanes Cedeño \\ Juan Rocha-Hoyos \\ Paolo Salazar Alvear \\ Johanna Medrano Barboza \\ Universidad Internacional SEK, Ecuador
}

Autor para correspondencia: antonio.1lanes@uisek.edu.ec

Fecha de recepción: 13 de abril de 2017 - Fecha de aceptación: 30 de Junio de 2017

Resumen: Los gases de combustión generados por el uso de combustibles fósiles, son la fuente principal de los problemas ambientales de la actualidad; el sector del transporte es a nivel mundial, uno de los principales precursores de daños ambientales, incidiendo significativamente en las ciudades más pobladas. Los biocombustibles son una alternativa que ha despertado una especial atención en gobiernos e instituciones por la significativa reducción de emisiones nocivas emanadas de los motores de combustión interna. El Ecuador, es uno de los países de América que no cumplen con los parámetros de calidad del aire dado por la Organización Mundial de la Salud, donde el transporte es el sector de mayor incidencia; los biocombustibles y sus mezclas son una alternativa para minimizar estos daños al ambiente y a la salud. Este trabajo tuvo como objetivo realizar una valoración del uso del biodiesel en el Ecuador, mediante la revisión documental desde sus materias primas, producción y uso para la recopilación de evidencias y evaluación de posibilidades de implementación de los biocombustibles a mayor escala. Se puede constatar que el mismo puede ser obtenido de diversas fuentes, y que su empleo en MCI en forma de mezclas con diésel, permite una disminución de las emisiones.

Palabras claves: ecuador; biocombustibles; emisiones; biodiesel; transesterificación

\begin{abstract}
Combustion gases generated by fossil fuels are the principal source of environmental problems now a days; transport sector is worldwide, one of the main cause of environmental damage, primarily in populated cities. Biofuels are an alternative that has awake the attention of governments and institutions due to the significant decrease of harmful emission of inter combustion engines. Ecuador is an American country that does not meet air quality parameters established by the World Health Organization; transport sector contributes to these emissions; biofuels and their mix with other fuels are an alternative to minimize environmental troubles and health problems. This investigation had as an objective to make a valuation of biodiesel uses in Ecuador, by the documentary revision from raw materials and production chain to the final uses to collect evidence and evaluate the implementation of biofuels at bigger scale. Can be seen that biodiesel is obtained from different sources, and its use in inter combustion engines as biodiesel mix, leads to a reduction of emissions.
\end{abstract}

Key words: ecuador biofuels; emissions; biodiesel; transesterification 


\section{Introducción}

Con las crisis recurrentes de los precios del petróleo a nivel internacional y las consecuentes repercusiones en las economías de los países en vías de desarrollo, la búsqueda de productos capaces de reducir la dependencia del petróleo es una tarea importante y urgente.

En los últimos años, debido al déficit petrolero y la contaminación que genera el uso de combustibles derivados del petróleo, el hombre ha buscado fuentes de energía alternas al uso de este hidrocarburo, atendiendo al potencial de otros recursos naturales (Fukuda et al., 2001). Una de esas alternativas es el biodiesel, que no es más que un metil éster consistente en ácidos grasos de cadena larga procedente de aceites vegetales comestibles o no comestibles, grasas animales y residuos de grasas usadas en restaurantes (Issariyakul \& Dalai, 2014).

Las ventajas más evidentes del biodiesel es que se trata de una sustancia biodegradable, no explosiva, no inflamable, renovable, no tóxica, cuya combustión genera bajos niveles de emisiones en gases de efecto invernadero como $\mathrm{CO} 2, \mathrm{NOx}, \mathrm{SOx}$ y material particulado (MP) (Mofijur et al., 2016); además, en comparación con el diésel obtenido a partir de combustibles fósiles, posee mayor número de cetano, punto de inflamabilidad y mejores características lubricantes sin variantes en el calor de combustión (Mahesh et al., 2015; DI et al., 2009). Sin embargo, para poder ser usado como combustible requiere de tratamientos para disminuir su alta viscosidad, su alto contenido de ácidos grasos que pueden formar gomas, depósitos de carbono y/o polimerizar durante el proceso de combustión y almacenamiento (Fukuda et al., 2001).

El biodiésel ha demostrado ser un buen combustible en motores de combustión, al ser mezclado en cualquier proporción con diésel proveniente de combustibles fósiles (Mofijur et al., 2015). Hasta la fecha, tres procesos han sido estudiados para hacer del biodiésel un combustible viable, a saber: pirólisis, micro-emulsificación y transesterificación. Entre ellos, la transesterificación ha demostrado ser un proceso sencillo y con mejores rendimientos. Se trata de un proceso catalizado o no, en el que un aceite y un alcohol reaccionan para producir un éster alquílico de ácidos grasos (biodiésel) y glicerina. De los alcoholes comerciales, el metanol es el más ampliamente usado debido a su disponibilidad y bajo costo (Wang et al., 2006).

\section{Revisión de la literatura}

\section{Panorama del escenario de emisiones}

\section{Análisis de las emisiones a partir del biodiésel}

Diferentes estudios han demostrado que el biodiésel reduce substancialmente la emisión de la mayoría de gases y partículas contaminantes de la atmósfera (ver tabla 1). Al ser un combustible oxigenado, el biodiésel tiene una combustión más completa que el diésel, mejorando por eso la composición de las emisiones.

\section{Tabla 1-Variación de las emisiones contaminantes del biodiésel vs diésel convencional Agente contaminante de la atmósfera Variación de emisiones}




\begin{tabular}{lll} 
& $\begin{array}{l}\text { Durante la } \\
\text { combustión } \\
(\%)\end{array}$ & $\begin{array}{l}\text { Total } \\
(\%)\end{array}$ \\
\hline $\mathrm{CO}$ & -46 & -35 \\
$\mathrm{CH}_{4}$ & 0 & -3 \\
$\mathrm{~N}_{2} \mathrm{O}$ & 0 & -66 \\
$\mathrm{Hidrocarburos}\left(\right.$ sin incluir $\left.\mathrm{CH}_{4}\right)$ & -37 & 238 \\
Hidrocarburos (no especificados) & 0 & -39 \\
Hidrocarburos policíclicos aromáticos & & -80 \\
Hidrocarburos policíclicos aromáticos nitrogenados & & -90 \\
Benceno & 0 & -96 \\
Formaldehído & 0 & -96 \\
PM10 & -68 & -45 \\
Partículas no especificadas & 0 & -25 \\
$\mathrm{SO}_{\mathrm{X}}$ & -100 & -8 \\
$\mathrm{NO}$ & 9 & 13 \\
$\mathrm{HCL}$ & 0 & 14 \\
$\mathrm{HF}$ & 0 & -16 \\
\hline
\end{tabular}

Fuente: Sheehan et al. (1998) \& Beer et al. (2002)

El biodiesel reduce las emisiones de partículas sólidas menores a 10 micrones (PM10), monóxido de carbono ( $\mathrm{CO}$ ) y óxidos de azufre ( $\mathrm{SOx}$ ), peligrosos agentes contaminantes. La Environmental Protection Agency (EPA) en un estudio compilatorio de diversas investigaciones sobre emisiones vehiculares con biodiesel, concluyó que las emisiones vehiculares de material particulado se reducían en un $47 \%$ cuando se usaba biodiésel, y las de monóxido de carbono en un $48 \%$. Sheehan et al. (1998), utilizando biodiésel de soya en autobuses de transporte urbano, observaron que las emisiones a lo largo del ciclo de vida del biodiésel se reducían en $44 \%, 35 \%$ y $8 \%$ para PM10, CO y SOx, respectivamente.

En el caso de las emisiones durante la combustión, las reducciones eran mucho más significativas: 68 \% para las PM10, 46 \% el CO y $100 \%$ los SOx, ya que el biodiésel no contiene azufre. Estudios realizados para el National Renewable Energy Laboratory de los Estados Unidos encontraron que las emisiones de material particulado dependen del contenido de oxígeno del combustible (Graboski et al., 2003). Analizando las emisiones de biodiésel proveniente de diferentes materias primas, se hallaron que todos reducen la emisión de partículas en comparación con el diésel. Para biodiésel con un índice de cetano mayor a 45 aproximadamente, la reducción en PM fue proporcional al contenido de oxígeno. Para biodiésel con número de cetano menor a 45, la reducción fue menor.

Asimismo, comparando ésteres metílicos y etílicos de los mismos ácidos grasos, Graboski et al. (2003) no encontraron diferencias consistentes en sus emisiones. Los índices de peróxido y de acidez, así como el contenido de glicerina del biodiésel tampoco tuvieron ningún efecto sobre las emisiones reguladas (CO, NOx, SO2, HCs totales, PM) en este estudio.

En el caso de los hidrocarburos (HC), si bien el biodiésel produce mayor cantidad en su ciclo de vida, durante la combustión las emisiones disminuyen en un $37 \%$ (Sheehan et al., 1998). El estudio de la EPA, encontró que las emisiones de HC durante la combustión disminuyen un $67 \%$. Las emisiones del biodiésel también tienen niveles menores de hidrocarburos policíclicos aromáticos (HPA, posibles cancerígenos), debido a que el biodiésel no contiene compuestos aromáticos de ningún tipo (Beer et al., 2002; Lin et al., 2006). 
También se pueden observar significativas reducciones en los compuestos aldehídos, especialmente el formaldehído y el acetaldehído. En lo que respecta al benceno, las emisiones se reducen prácticamente en un $95 \%$ en todo el ciclo de vida del biodiésel, con una combustión totalmente libre de este compuesto cancerígeno (Sheehan et al., 1998).

El biodiésel prácticamente no es tóxico en caso de ingestión (en peces o mamíferos). La concentración de biodiésel para que llegue a ser letal por ingestión oral es muy elevada, alrededor de $17,4 \mathrm{~g} / \mathrm{kg}$ de peso corporal, lo cual significa que una persona de $80 \mathrm{~kg}$ tendría que tomar alrededor de 1,6 L de biodiésel para que tenga efectos mortales. La sal común $(\mathrm{NaCl})$ es aproximadamente diez veces más tóxica. El impacto en la salud humana es un criterio importante cuando se considera la idoneidad de un combustible para aplicaciones comerciales.

En cuanto a la toxicidad acuática, según el National Institute for Occupational Safety and Health (NIOSH) de los Estados Unidos, ésta es muy baja. Se requieren concentraciones altísimas en el agua, mayores a $1000 \mathrm{mg} / \mathrm{L}$, para llegar a niveles letales. Por ello el biodiésel es bastante inofensivo para la fauna acuática.

Además, el biodiésel es altamente biodegradable en el agua. En estudios de la Universidad de Idaho se encontró que el biodiésel se degrada a un ritmo muy superior al del diésel convencional e incluso tan rápido como la dextrosa (azúcar). En una prueba en solución acuosa, a los 28 días se había degradado el $95 \%$ del biodiésel, mientras que el diésel convencional se había degradado en un $40 \%$. En una segunda prueba, esta vez en ambientes acuáticos, el $87 \%$ del biodiésel se degradó en 28 días, mientras que la degradación del diésel sólo fue del orden del $26 \%$.

La mezcla de biodiésel con diésel o con gasolina incrementa la biodegradabilidad del combustible, debido a efectos sinérgicos de «cometabolismo». Así, el tiempo necesario para alcanzar un $50 \%$ de biodegradación se reduce de 28 a 22 días en el caso del B5 (mezcla de $5 \%$ de biodiésel y $95 \%$ de diésel) y de 28 a 16 días en el caso del B20 (Pasqualino et al., 2006). Estos efectos sinérgicos son importantes por dos razones:

El biodiésel se comercializa actualmente de manera principal mezclado con diésel, y los riesgos de derrame son los mismos que para el diésel puro.

El biodiésel podría ser utilizado como un «acelerador» de la biodegradación en caso de derrames de hidrocarburos en medios acuáticos.

Asímismo, el biodiésel es menos tóxico y más biodegradable que el diésel en el suelo. En un estudio realizado, se encontró que el combustible diésel es tóxico a una concentración de $3 \%$ en peso en el suelo, mientras que el biodiésel no muestra toxicidad hasta concentraciones de 12 $\%$ en peso (esta fue la máxima concentración probada en el estudio). Igualmente, se observó que el biodiésel es más fácilmente degradado por la microbiota del suelo: mientras que $80 \%$ del biodiésel fue completamente biodegradado, sólo $61 \%$ del diésel lo fue (Lapinskienė et al., 2006). Estas características también señalan al biodiésel como combustible apropiado en zonas agrícolas o rurales donde la contaminación del suelo por derrames es más frecuente. 


\section{Contribución del sector del transporte a la emisión de CO2 en el Ecuador}

El uso de combustibles fósiles en el Ecuador ha estado en constante aumento en las recientes décadas según informe del Ministerio Coordinador de Sectores Estratégicos (Delgado, 2015), siendo el petróleo la fuente con una mayor contribución como se muestra en la figura 1. En particular, su uso en vehículos ha crecido tanto por el aumento del parque automotor como por el hecho que los combustibles fósiles presentan un subsidio en el país. Los principales combustibles en motores vehiculares en Ecuador provienen del petróleo fósil y son la gasolina (en vehículos livianos) y el diésel (en vehículos pesados), en la figura 2, se representa la demanda de energía por sectores (con base al 2014), donde el transporte posee el $42 \%$ de la demanda, coincidiendo con el comportamiento a nivel mundial donde el transporte representa el $59 \%$ con un pronóstico en su crecimiento al $63 \%$ para el 2040 (IEA, 2013).

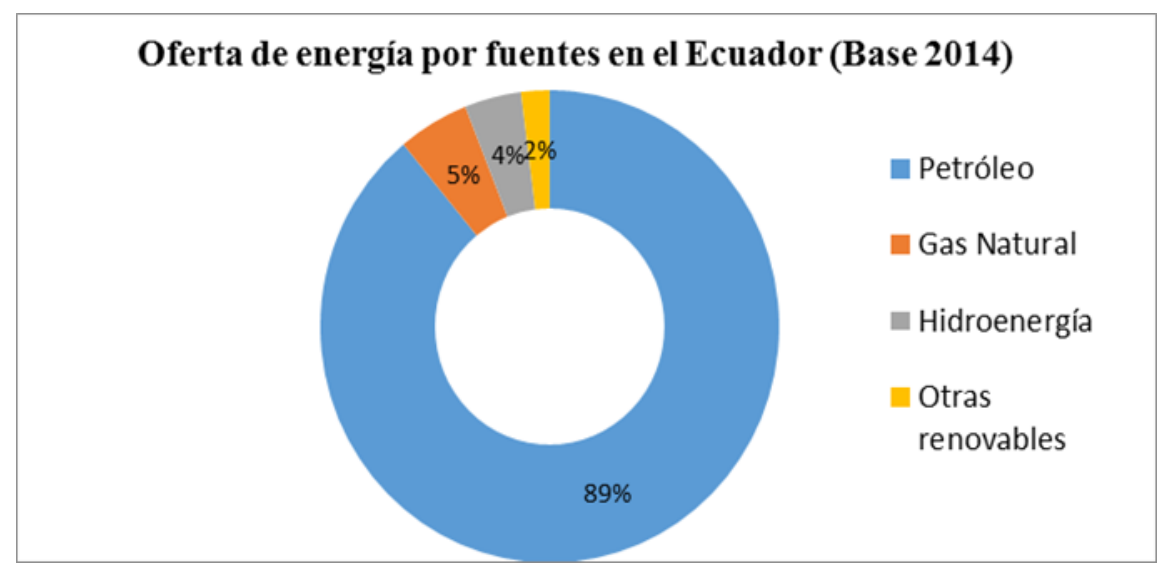

Figura 1. Porcentaje de contribución de las fuentes energéticas a la matriz energética del Ecuador (Delgado, 2015).

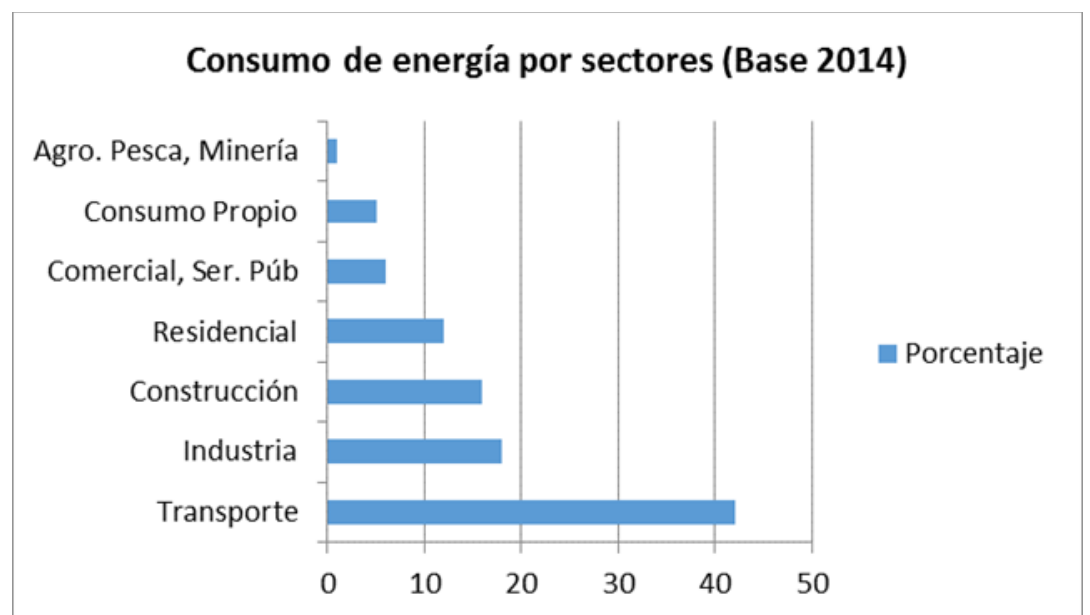

Figura 2. Demanda de energía por sectores en el Ecuador (Delgado, 2015).

Las emisiones de $\mathrm{CO} 2$ han tenido una tendencia al incremento como se muestra en la figura 3 según el World Bank (2011). Según Alcántara \& Padilla (2005), este crecimiento tiene asociado múltiples factores tales como: El desarrollo económico, crecimiento demográfico, 
cambio tecnológico, dotación de recursos, estructuras institucionales, modelos de transporte, estilos de comercio internacional, etc. La identificación del tipo de fuentes de emisiones de CO2 y de su magnitud es información esencial para la planificación económica y la toma de decisiones.

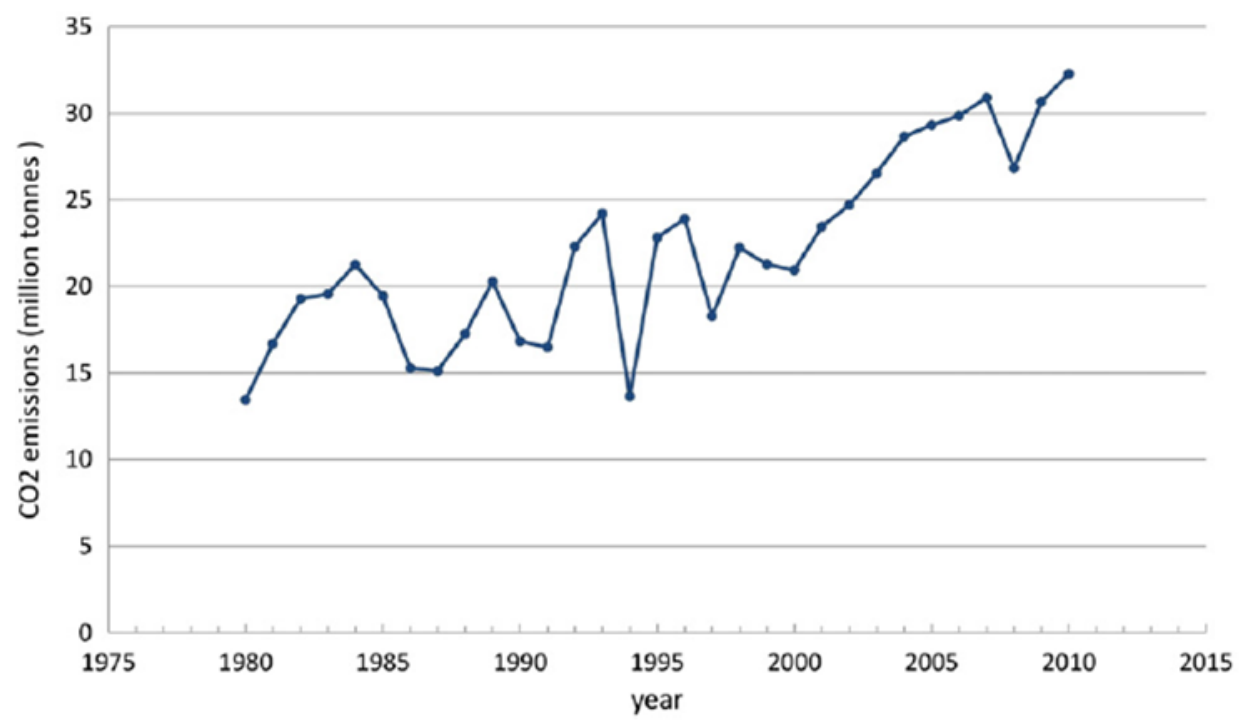

Figura 3. Ecuador. Emisiones de CO2 (1980-2010).

Trabajo realizado por Robalino-López et al., (2014) aplicando cuatro escenarios posibles, determina las emisiones de CO2 en función del tiempo para el período 2011-2020, dando como resultado en todos los casos un incremento del CO2 (figura 4).

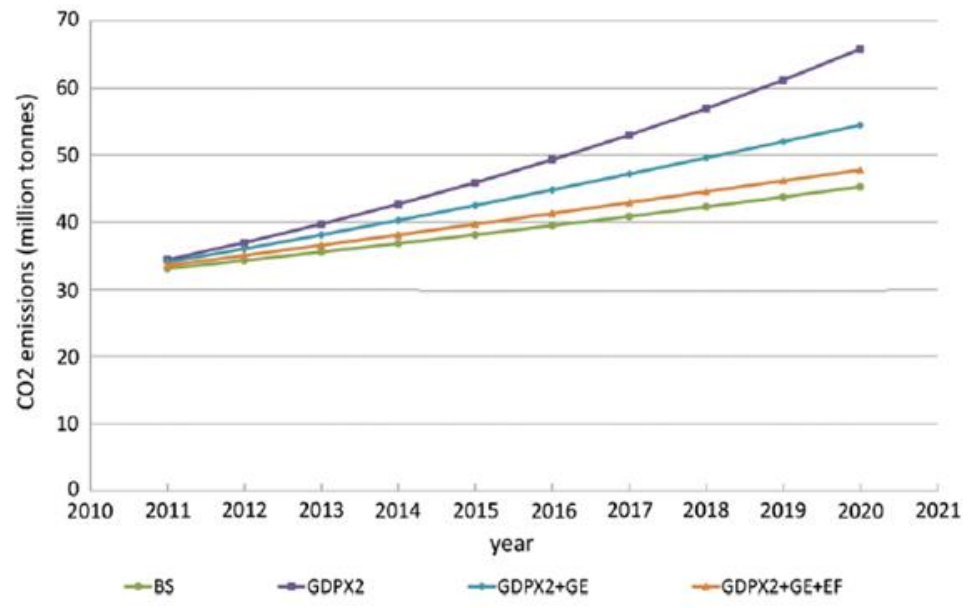

Figura 4. Emisiones de CO2 en el Ecuador para el período 2011-2020 (Robalino-López et al., 2014).

Según el Balance Nacional 2015 (Delgado, 2015) realizado por el Ministerio Coordinador de Sectores Estratégicos (con base 2014), contabiliza que las emisiones de gases de efecto invernadero (GEI) (dióxido de carbono -CO2-, metano -CH4 y óxido nitroso - N2O) incrementaron un 10,7 \% respecto al 2013. Lo que representó la emisión de 45,8 millones de toneladas de $\mathrm{CO} 2$ equivalentes por parte del sector energético del país, de las cuales el transporte es el mayor generador de gases, ocupando el $39 \%$ del total de emisiones. La demanda de energía 
se incrementa constantemente en todos los sectores, siendo el sector del transporte el de mayor significación alcanzando 40,6 \% en 2000, 43,6 \% en el 2013 y $42 \%$ en 2014, manifestando en sentido general desde el 2004 - 2014, un crecimiento promedio anual del 4,3\%.

Dentro de la actividad del transporte, el terrestre demanda el $86 \%$ del total de energía, muy por encima del marítimo con un $8 \%$ y el aéreo con el $6 \%$. Dentro del transporte terrestre, el de carga pesada y liviana, son los que más energía consumen con un $42 \%$ y $18 \%$, respectivamente (figura 5), trabajo desarrollado por Sierra (2016), muestran resultados similares reportados por el Ministerio Coordinador de Sectores Estratégicos, solo que en su caso la carga liviana y pesada, son los que más energía consumen con un $42 \%$ y $22 \%$, respectivamente.

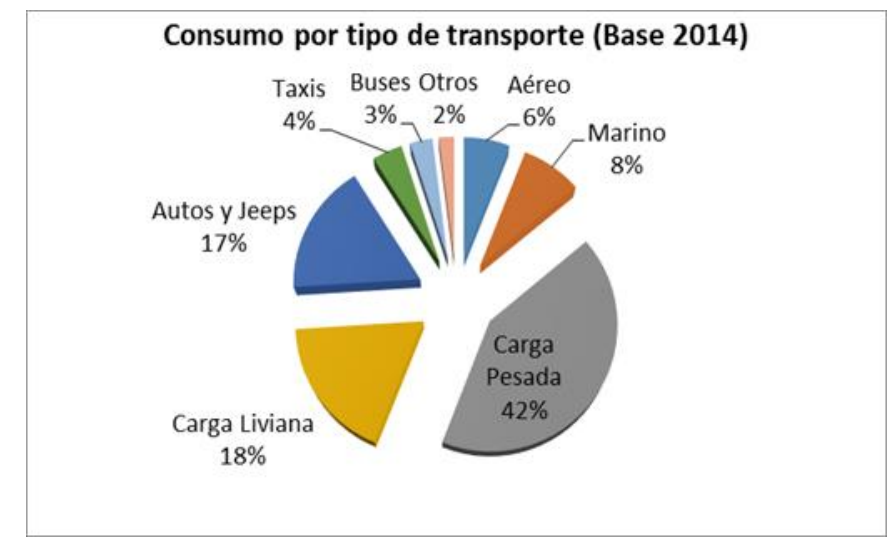

Figura 5. Demanda de energía por tipo de transporte en el Ecuador (IEA, 2013).

El sector del transporte es el que mayor influencia posee en la emisión de los Gases de Efecto Invernadero (GEI) con un $45 \%$, seguido de la industria y las centrales eléctricas, ambas con un $13 \%$ (figura 6 ).

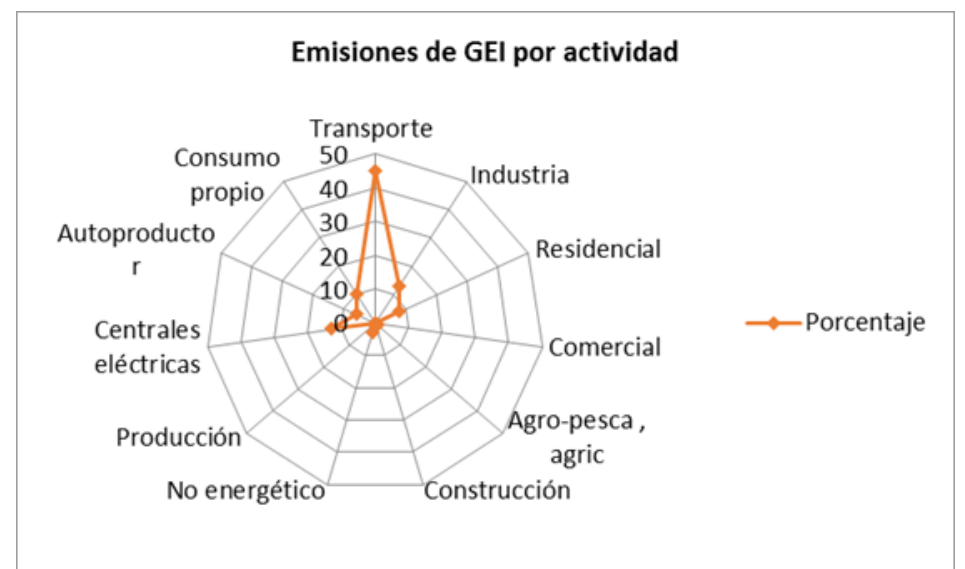

Figura 6. Comportamiento en la emisión de GEI por actividad (Tandazo, 2015).

La salud y el entorno ambiental de la emisión de combustibles

Las emisiones de gases de escape contribuyen a la lluvia ácida y a la acumulación mundial de CO2. Cada uno de ellos tiene amplias consecuencias para la salud humana. En los países desarrollados, el escape de tráfico representa aproximadamente un cuarto de todas las emisiones de CO2 (McMichael, 2000). 
El biodiesel es un combustible oxigenado (de ahí una combustión más completa) y provoca una menor formación y emisión de partículas. La opacidad del humo es una medida directa de humo y hollín. Varios estudios muestran que la opacidad del humo para el biodiesel es generalmente menor según: Agarwal (1998); Masjuki (1996); Kalligeros et al. (2003) \& Ceviz et al. (2011).

Varias investigaciones experimentales se realizan en motores diésel DI de cuatro tiempos con ésteres metílicos de aceites vegetales y encontraron que las emisiones de hidrocarburos son mucho menores en el caso del biodiesel comparado con el diésel (Kalligeros et al., 2003; Agarwal, 1998).

El CO es un producto tóxico de la combustión resultante de la combustión incompleta de hidrocarburos. En presencia de suficiente oxígeno, $\mathrm{CO}$ se convierte en $\mathrm{CO} 2$. El biodiesel es un combustible oxigenado y conduce a una combustión más completa, por lo que las emisiones de CO se reducen en el escape. Altin et al. (2001), informó que la emisión de CO para el biodiésel es ligeramente más alta en comparación con el diésel (Agarwal, 2007).

Partículas (PM) es el término genérico utilizado para un tipo de contaminantes atmosférico, ya que pueden absorber y transferir una multitud de contaminantes. Sin embargo, sus componentes principales son metales, compuestos orgánicos, material de origen biológico, iones, gases reactivos y el núcleo de partículas de carbono. El tamaño de las partículas varía (PM2, 5 y PM10 para el diámetro aerodinámico menor que 2,5 mm y $10 \mathrm{~mm}$ respectivamente), y esto hace que sean peligrosas, como las de tamaños de partículas grandes (partículas gruesas), que son fuertes en términos de mortalidad y efectos cardiovasculares y respiratorios (Kampa \& Castanas, 2008; Rocha-Hoyos et al., 2017).

El biodiesel muestra una clara reducción de hollín y menor potencia mutagénica de la PM es clara. Estas ventajas se deben probablemente a la ausencia de azufre y aromáticos. Una desventaja es el aumento de la citotoxicidad observada en el modo de carga en vacío (ralentí), según Siegel et al. (1987) \& Bünger et al. (2000).

\section{Obtención del biodiesel}

\section{Materias Primas}

Existe una gran diversidad de recursos que pueden ser usados como materias primas para la obtención de biodiésel, entre ellos los aceites vegetales de palma, soya, coco, girasol, canola, jatropha, moringa oleífera, así como semillas de algodón, de albaricoque, de nim; entre otros, siempre y cuando no haya competencia con la alimentación humana. También, las grasas animales de aves, cerdos y aceites de pescado han sido empleadas para obtener biodiesel, pero con el impedimento de ser más viscosas y existir en fase sólida a temperatura ambiente debido a la gran cantidad de ácidos grasos saturados. Otra fuente importante de biomasa para la obtención de biodiésel son los aceites obtenidos a partir de algas, bacterias y hongos, debido a su rápido crecimiento (Xiao et al., 2009). Los aceites residuales de cocina, principalmente proveniente de frituras (en lugar de usar aceites vírgenes de mayor costo), se muestran como una materia prima 
adecuada para la producción de biodiésel, debido a su abundancia en todo el mundo; además, de costar la mitad del precio de su homólogo virgen (Wang et al., 2007), lo cual reduciría los costos de producción de biodiésel, y se daría solución a un problema de contaminación por disposición de este recurso (Kulkarni \& Dalai, 2006). En la tabla 2, se relacionan algunos países del continente americano y las principales fuentes de biodiésel.

Tabla 2 Fuente: Mofijur et al. (2016).

\begin{tabular}{ll}
\hline Países & Materia prima potencial \\
\hline $\begin{array}{l}\text { Argentina } \\
\text { Brasil }\end{array}$ & $\begin{array}{l}\text { Habas de Soja } \\
\text { Habas de soja / aceite de palma/ aceite de castor/ aceite } \\
\text { de algodón }\end{array}$ \\
Cuba & Moringa/ Nim/ Jatropha \\
Ecuador & Grasa animal/ aceite usado de cocina/ Palma \\
México & Grasa animal/ aceite usado de cocina \\
Perú & Aceite de palma/ jatropha \\
\hline
\end{tabular}

\section{Procesos de obtención del biodiésel}

Independientemente de cual sea el origen de la materia prima (vegetal o animal) para la obtención del biodiésel, este no puede ser usado directamente en motores de combustión debido a su alta viscosidad, baja volatilidad y su carácter poliinsaturado que le hace formar gomas o polimerizar a altas temperaturas (Mofijur et al., 2016). Los métodos empleados actualmente para la reducción de la viscosidad son la mezcla de biodiésel con diferentes combustibles fósiles (como diésel o nafta), la pirólisis, la micro-emulsificación y la transesterificación, como se mencionó anteriormente.

Entre ellos, el proceso de transesterificación consta de una reacción reversible y consecutiva de tres etapas, en la que reaccionan los triacilglicèridos del aceite (animal o vegetal) con exceso de un alcohol de cadena corta (como el metanol). La reacción puede ser catalizada por un álcalis, una base, enzimas o no emplear ningún catalizador, para obtener diglicéridos y monoglicéridos, como compuestos intermediarios. Tres moles de biodiesel y un mol de glicerina se obtienen por cada mol de triacilglicèridos (Mofijur et al., 2016). Las ecuaciones 1, 2 y 3, muestran las reacciones involucradas:

$$
\begin{aligned}
& \text { Triglicéridos }(\mathrm{TG})+\mathrm{ROH} \leftrightarrow \text { Diglicéridos (DG)+RCOOR_1 (alquil éster) } \\
& \text { Diglicéridos (DG)+ROH } \leftrightarrow \text { monoglicéridos (MG)+RCOOR_2 (alquil éster) }
\end{aligned}
$$

$$
\text { Monoglicérido }(\mathrm{MG})+\mathrm{ROH} \leftrightarrow \text { Glicerina+RCOOR_3 (alquil éster) }
$$

Aunque la obtención de biodiésel a partir de la transesterificación ha sido usada en escala industrial por décadas, presenta algunas desventajas asociadas a la remoción de catalizador, altos requerimientos de energía, la recuperación de la glicerina, reacciones secundarias (principalmente de saponificación) y alto consumo de agua (Zhang et al., 2003). Hoy en día, las investigaciones apuntan a la obtención de biodiésel empleando lipasas como catalizadores para remediar algunas de las desventajas anteriormente mencionadas. 
Las reacciones de transesterificación pueden llevarse a cabo sin catalizador o con catalizadores homogéneos o heterogéneos. La síntesis de biodiésel no catalítica, requiere de temperaturas mayores a $350^{\circ} \mathrm{C}$, valores muy altos y riesgosos considerando que los ésteres se degradan a los $400{ }^{\circ} \mathrm{C}$ (Xiao et al., 2009). La catálisis homogénea de la transesterificación es un método fácil, pero de separación compleja del producto, ya que se obtiene una mezcla que requiere de varias etapas de purificación. También, tiende a la formación de jabón por lo que requiere de grandes cantidades de agua de lavado; todo esto se traduce en costos. Por su parte, la catálisis heterogénea de la reacción de transesterificación de aceites de cocina usados, es más económica y menos compleja. Luego de la reacción, el catalizador se encuentra en una fase diferente a la de los reactantes no consumidos y productos, por lo que su separación es simple y se reduce significativamente la formación de jabones (Wang et al., 2007; Luković et al., 2011). $\mathrm{El}$ óxido de calcio $(\mathrm{CaO})$ es uno de los mejores catalizadores heterogéneos, gracias a su alta basicidad, bajo costo, baja solubilidad (por lo que puede recuperarse) y fácil manejo en comparación con un catalizador homogéneo (Mahesh et al., 2015).

\section{Objetivos de la producción de biodiesel}

En la actualidad, los países están mirando y trabajando hacia la obtención de combustibles más limpios como una opción de desarrollo de la economía, la seguridad energética y como acción de gestión para la remediación de daños ambientales causados por el uso de combustibles fósiles. Los biocombustibles, son un mecanismo para reducir las emisiones de CO2 y otros gases de efecto invernadero; además, al dirigir la producción agrícola hacia la obtención de materias primas para la obtención de biocombustibles, se fortalece la agricultura, se generan fuentes de empleo y de manera directa o indirecta se disminuye con la pobreza del sector aledaño a los cultivos (Mofijur et al., 2016). Considerando el factor de la sustentabilidad, el desarrollo de los biocombustibles a partir de materias primas agrícolas como maíz, caña de azúcar y palma aceitera, ha tenido un crecimiento exponencial en los últimos diez años, especialmente en la Unión Europea, Australia, y en el continente americano destacan Estados Unidos, Brasil y Argentina.

En Brasil, la producción de biocombustibles, específicamente de bioetanol data de más de treinta años, y hoy en día esta industria del etanol obtenido a partir de caña de azúcar desarrollada en Brasil es mucho más eficiente que la industria estadounidense basada en insumos de maíz, al punto que para la fecha, no existen combustibles derivados del petróleo en las estaciones de servicio (BuDny \& Sotero, 2007). Según información de la Organización Latinoamericana de Energía, OLADE; en el año 2010 en Ecuador se inició un plan piloto para la producción de gasolina mezclada al $5 \%$ con etanol (obtenido a partir de caña de azúcar y palma aceitera); dicho proyecto se denominó Ecopaís. Hoy en día existen 43 estaciones de servicio en Guayaquil, que suministran esta mezcla de combustible. Para el año 2013, el gobierno dio pie a otro plan para que el diésel de origen fósil que consume el parque automotor ecuatoriano, se mezclará en un $5 \%$ con biodiésel, y que esta proporción se incrementará hasta alcanzar el 10 $\%$, atendiendo a que la producción de biodiésel estimula la producción agrícola y genera beneficios ambientales. El biodiésel puede mezclarse con el diésel de origen fósil, y dependiendo de las concentraciones de mezclado, puede usarse en el parque automotor sin requerir modificaciones en los motores (Paredes, 2015). 


\section{Cronología del desarrollo mundial de biocombustibles}

Para la Unión Europea (UE), el desarrollo de la bioenergía es una prioridad. En términos de la Comisión Europea, el incremento de los biocombustibles en su balance energético fortalecerá la sustentabilidad económica a través de la reducción de la dependencia del combustible importado, que actualmente es del $50 \%$ (con proyecciones de crecimiento del $70 \%$ en ausencia de medidas). Por otro lado, consideran que el desarrollo de esta nueva industria también jugará un rol importante en la generación de nuevos puestos laborales en el sector de la pequeña y mediana empresa y en la reducción de las emisiones de CO2 conforme a los compromisos asumidos en el Protocolo de Kyoto.

El gobierno de los Estados Unidos, tanto a nivel federal como estatal, ha implementado una serie de medidas para el desarrollo de las fuentes alternativas de energía, principalmente del etanol. Su motivación fue la subida de los precios internacionales de los combustibles fósiles y la dependencia del petróleo importado.

De este modo, la seguridad energética y el abastecimiento es lo que impulsa, en primer lugar, el desarrollo de esta industria, seguido por los objetivos ambientales. Casi la totalidad de la producción de biocombustibles es de etanol, del cual el $95 \%$ se elabora a partir del maíz, mientras que el $5 \%$ restante deriva del sorgo, la cebada, el trigo y la papa. Según estimaciones del Departamento de Agricultura (USDA), recogidas en Yacobucci et al. (2007), cerca de 2.200 millones de toneladas de maíz deberían haber sido utilizados para producir 6.000 millones de galones de bioetanol durante la campaña de comercialización del maíz 2006/2007. Esto implica utilizar casi el $20 \%$ de la producción de maíz estimada para este ciclo. Como el etanol representa $2,4 \%$ de las naftas para automotores -medida en energía equivalente-, el empleo total de la producción de maíz para etanol sólo alcanzaría para reemplazar poco más del $10 \%$ de las naftas consumidas y alrededor del $20 \%$ del petróleo importado (Yacobucci et al., 2007).

Tanto en los Estados Unidos como en la Unión Europea, la política oficial promovió el desarrollo del bioetanol y del biodiesel mediante distintas medidas interrelacionadas, además del estándar de mezcla obligatoria. Estas medidas son necesarias para sostener la producción en estas dos economías, dado que el costo de producción del etanol en Estados Unidos es superior al precio de la nafta y el costo del biodiesel en la UE es mayor al precio del diésel-oil (Galperín \& Llana, 2009).

En Ecuador existen múltiples oportunidades alrededor del desarrollo de biocombustibles de segunda generación, pero se requiere ser ambicioso en la inversión, investigación y desarrollo de plantas piloto. Por ello, en lugar de invertir en una profundización masiva de la producción agrícola de biocombustibles de primera generación, Ecuador puede ganar mucho más invirtiendo en una industria que utiliza residuos ya existentes y en generación de conocimiento y valor agregado. Una política de este tipo es un paso concreto para la construcción de un país pospetrolero y pos-agrícola primario (Castro, 2012).

\section{Estándares mundiales de biocombustibles}


Hay plantas con propiedades similares a las de los derivados del petróleo que, mediante un sencillo tratamiento, pueden proporcionar combustibles líquidos. En este sentido pueden señalarse la palma africana (Elaeis guineensis), la tabaiba (Euphorbia lathyris), etc.

En el Ecuador se utilizó la higuerilla (Ricinus communis), de la que se extrae el aceite de castor. La fabricación de biodiesel en Ecuador a nivel industrial se hace a partir principalmente de la palma africana (Elaeis guineensis), su fabricación está regulada por la norma ASTM D6751.

El combustible biodiesel tiene en general, propiedades iguales o similares del combustible diésel convencional y puede mezclarse en cualquier proporción con el combustible diésel (Coronado et al., 2009). En la actualidad se han llevado a cabo numerosas investigaciones referidas a la obtención de biodiesel a partir de diferentes fuentes, trabajos como los de Jansri (2015), López et al. (2015), Saba et al. (2016), entre otros, y evaluado en motores de combustión interna (MCI) destacándose los trabajos de Badruddin et al. (2015) \& y Can et al. (2016).

\section{Operación del motor}

Un motor diésel de inyección directa de un solo cilindro probado con gasóleo regular, biodiesel limpio con inyección de butanol en el colector de admisión. Un motor alimentado con biodiesel limpio muestra una reducción del $5 \%$ de la eficiencia de conversión de combustible y un aumento del $17 \%$ en el consumo específico de combustible con respecto al gasóleo, además el motor diésel puede operar en el biocombustible (biodiésel más alcohol) con la eficiencia de conversión del combustible equivalente a la del aceite diésel (Micklich et al., 2016).

El biodiésel producido a partir de aceite de avellana donde los porcentajes de biodiésel que se probaron fueron: $20 \%$ (B20), $40 \%$ (B40), $60 \%$ (B40) y $80 \%$ (B80). Además, se probó el combustible diésel puro, donde muestra las características de rendimiento del motor. A partir de estas cifras se nota que hay un aumento en el par motor y la potencia de frenado con el aumento progresivo de la velocidad del motor. Pero también hay una ligera caída en el par y la potencia de frenado con las mezclas a baja velocidad del motor. Cuando la velocidad del motor aumenta más allá de $1.750 \mathrm{rpm}$, la potencia obtenida es en un 3\% menor (Ceviz et al., 2011). El par y la potencia se relacionan con la densidad de energía del combustible, y hay un gran número de estudios que indican la pérdida de potencia con el uso de combustible mezclado con biodiésel (Canakci, 2007; Graboski \& McCormick, 1998).

Las operaciones de B20 presentan los mejores resultados para el rendimiento del motor y las emisiones de escape. En esta mezcla las emisiones de $\mathrm{CO}$ y el consumo específico de combustible disminuyeron en aproximadamente 7.1 y $8.2 \%$, respectivamente, en comparación con los experimentos con diésel puro. La eficacia del motor se incrementó en un 13,7\% (Cerviz et al., 2011).

En un motor mono cilíndrico, 4T, inyección directa y enfriado por aire se aplicó biodiesel de palma y de jatrofa para determinar que mezcla de biodiesel D90PB5JB5 (es decir, el $90 \%$ de diésel y $10 \%$ de biodiésel) muestra incremento medio del 4,65 \% en la potencia al freno que el diésel. Hubo una ligera disminución observada para mezclas inferiores. D60JB20PB20 y 
D20JB40PB40 (es decir, el $20 \%$ de diésel y $80 \%$ de biodiésel) han mostrado hasta aumento medio del $15 \%$ en la eficiencia térmica del freno (Nalgundwar et al., 2016).

Se analizaron las propiedades de los combustibles diésel $\mathrm{N}^{\circ} 1 \mathrm{y} \mathrm{N}^{\circ} 2$ y el biodiésel producido a partir de aceite de soja puro (B100) y como mezcla al $20 \%$ con el combustible diésel $\mathrm{N}^{\circ} 2$ (B20) en un motor diésel alimentado con estos combustibles. Los resultados del estudio mostraron que hubo un aumento en el consumo específico de combustible. Sin embargo, el uso de combustible diésel No. 1 dio mejores características. Además, el período de retraso de encendido para el B100 fue más corto que para los combustibles diésel (Canakci, 2007).

\section{Emisiones}

En un motor de cuatro cilindros, cuatro tiempos $2200 \mathrm{cc}$, diésel de pre-combustión alimentado con mezclas de 20, 50 y $80 \%$ de combustible diésel-biodiésel, el B20 produjo la concentración más baja de $\mathrm{CO}$ para todas las velocidades del motor, B50 produjo la mayor concentración de $\mathrm{CO} 2$ que otros combustibles para todas las velocidades del motor, excepto a 2.000 rpm donde B20 proporcionó el más alto valor, B100, B80 y diésel puro mostraron más emisiones de $\mathrm{HC}$ que otros combustibles. Los combustibles de biodiésel y biodiésel-diésel combinados produjeron NOx más alto para varias velocidades de motor como se esperaba (Lin et al., 2007; Cedeño et al., 2017).

Un motor diésel de inyección directa de un solo cilindro probado con gasóleo regular, biodiésel limpio con inyección de butanol en el colector de admisión, se mejoraron las emisiones de los motores con biocombustible, excepto el monóxido de carbono y los hidrocarburos no quemados (Micklich et al., 2016); el biodiésel producido a partir de aceite de soja puro (B100) y como mezcla al $20 \%$ con el combustible (B20) las emisiones de NOx aumentan, y se disminuyen de forma significativa las emisiones de monóxido de carbono (CO), hidrocarburos (HC) y humo para B100 en comparación con el combustible B20. Sin embargo, el uso de combustible diésel No. 1 dio mejores características. Además, el período de retraso de encendido para el B100 fue más corto que para los combustibles diésel (Micklich et al., 2016).

Experimentando el biodiésel producido a partir de aceite de avellana, cuando el porcentaje de la relación de mezclas biodiésel-diésel supera en un $20 \%$, las emisiones de HC y $\mathrm{CO}$ mostraron una tendencia creciente debido al aumento de la viscosidad del combustible mezclado con biodiesel (Ceviz et al., 2011).

Con el biodiésel de palma y de jatrofa se observó una disminución notable en la temperatura del gas de escape durante la mayor parte de mezclas de biodiésel. Había 7,1\%, $17,7 \%$ y 14,5 \% de reducción promedio de las emisiones de CO con muestras D90JB5PB5, D80JB10PB10 y D70JB15PB15 (mezclas de biodiésel que contienen 10 \%, $20 \%$ y $30 \%$ de biodiésel), respectivamente, en comparación con diésel. Mezclas inferiores de muestras de biodiésel D90JB5PB5 y D80JB10PB10 mostraron un 5,3\% y un incremento medio del 9,2 \% en las emisiones de NOx, respectivamente, que el diésel (Ceviz et al., 2011; Cedeño et al., 2017).

\section{Conclusiones}


Existe un gran potencial en el Ecuador para la producción de biodiesel, fundamentalmente del aceite de Palma y residuos de aceite vegetal usado.

El transporte es uno de los sectores con mayor incidencia en los efectos dañinos al ambiente y a la salud humana, siendo el sector de carga pesada uno de los de mayor significancia.

Los estudios posteriores indican que el biodiésel es una alternativa que en mezclas con el diésel, pueden ser empleadas satisfactoriamente en los motores de combustión interna, reduciéndose en su mayoría las emisiones al ambiente.

El principal proceso de obtención del biodiésel es la transesterificación, en el cual se sigue investigando para aumentar el rendimiento.

Las propiedades de los biocombustibles obtenidos a partir de las diferentes materias primas, no difieren mucho y los procesos de obtención se encuentran estandarizados por normas específicas.

\section{Bibliografía}

Agarwal, A. K. (1998). Vegetable oils versus diesel fuel: development and use of biodiesel in a compression ignition engine. TIDE, 8(3), 191-204.

Agarwal, A. K. (2007). Biofuels (alcohols and biodiesel) applications as fuels for internal combustion engines. Progress in energy and combustion science, 33(3), 233-271.

Alcántara, V., \& Padilla, E. (2005). Analysis of CO2 and its explanatory factors in the different areas of the world. Technical report, Universidad Autónoma de Barcelona.

Altın, R., Cetinkaya, S., \& Yücesu, H. S. (2001). The potential of using vegetable oil fuels as fuel for diesel engines. Energy conversion and management, 42(5), 529-538.

Badruddin, I. A., Badarudin, A., Banapurmath, N. R., Ahmed, N. S., Quadir, G. A., Al-Rashed, A. A., ... \& Kamangar, S. (2015). Effects of engine variables and heat transfer on the performance of biodiesel fueled IC engines. Renewable and Sustainable Energy Reviews, 44, 682-691.

Beer, T., Grant, T., Williams, D., \& Watson, H. (2002). Fuel-cycle greenhouse gas emissions from alternative fuels in Australian heavy vehicles. Atmospheric environment, 36(4), 753-763.

BuDny, D., \& Sotero, P. (2007). The global dynamics of biofuels. Brazil Institute Special Report, $4(3), 8$.

Bünger, J., Krahl, J., Baum, K., Schröder, O., Müller, M., Westphal, G. ... \& Hallier, E. (2000). Cytotoxic and mutagenic effects, particle size and concentration analysis of diesel engine emissions using biodiesel and petrol diesel as fuel. Archives of toxicology, 74(8), 490-498. 
Can, Ö. Öztürk, E., Solmaz, H., Aksoy, F., Çinar, C., \& Yücesu, H. S. (2016). Combined effects of soybean biodiesel fuel addition and EGR application on the combustion and exhaust emissions in a diesel engine. Applied Thermal Engineering, 95, 115-124.

Canakci, M. (2007). Combustion characteristics of a turbocharged DI compression ignition engine fueled with petroleum diesel fuels and biodiesel. Bioresource technology, 98(6), 11671175.

Castro, M. (2012). Reflexiones en torno al desarrollo de los biocombustibles en Ecuador.

Cedeño, E. A. L., Rocha-Hoyos, J. C., Puebla, Y. G., Tipanluisa, L., \& Velastegui, G. (2017).

Análisis Comparativo de los Gases Residuales de la Combustión y Fallas en Motores Hyundai Modelo 9h21/32, En Estación De Generación Eléctrica Distribuida. Innova Research Journal, 2(3).

Ceviz, M. A., Koncuk, F., Küçük, Ö. Gören, A. C., \& Yüksel, F. (2011). Analysis of combustion stability and its relation to performance characteristics in a compression ignition engine fueled with diesel-biodiesel blends. Energy Sources, Part A: Recovery, Utilization, and Environmental Effects, 33(10), 990-1003.

Coronado, C. R., de Carvalho, J. A., Yoshioka, J. T., \& Silveira, J. L. (2009). Determination of ecological efficiency in internal combustion engines: The use of biodiesel. Applied Thermal Engineering, 29(10), 1887-1892.

Delgado, D. (2015). Balance Energético Nacional 2015 año base 2014, from Ministerio Coordinador de Sectores Estratégicos.

Di, Y., Cheung, C. S., \& Huang, Z. (2009). Experimental investigation on regulated and unregulated emissions of a diesel engine fueled with ultra-low sulfur diesel fuel blended with biodiesel from waste cooking oil. Science of the total environment, 407(2), 835-846.

Fukuda, H., Kondo, A., \& Noda, H. (2001). Biodiesel fuel production by transesterification of oils. Journal of bioscience and bioengineering, 92(5), 405-416.

Galperín, C., \& Llana, C. P. (2009). Desarrollo de los biocombustibles, interrelación de políticas y opciones de política comercial. Revista argentina de economía agraria, 11(1).

Graboski, M. S., \& McCormick, R. L. (1998). Combustion of fat and vegetable oil derived fuels in diesel engines. Progress in energy and combustion science, 24(2), 125-164.

Graboski, M. S., McCormick, R. L., Alleman, T. L., \& Herring, A. M. (2003). The effect of biodiesel composition on engine emissions from a DDC series 60 diesel engine. National Renewable Energy Laboratory (Report No: NREL/SR-510-31461). 
IEA Statistics. (2013). Excerpt from: Energy Balances of non-oecd Countries (2015 Edition). International Energy Agency, web site: https://www.iea.org/publications/ freepublications/publication/WorldTrends_NonOECD_countries2015.pdf.

Issariyakul, T., \& Dalai, A. K. (2014). Biodiesel from vegetable oils. Renewable and Sustainable Energy Reviews, 31, 446-471.

Jansri, S. (2015). Preparation of vegetable oil as biodiesel feedstock via re-esterification: a suitable catalyst. Energy Procedia, 79, 143-148.

Kalligeros, S., Zannikos, F., Stournas, S., Lois, E., Anastopoulos, G., Teas, C., \& Sakellaropoulos, F. (2003). An investigation of using biodiesel/marine diesel blends on the performance of a stationary diesel engine. Biomass and Bioenergy, 24(2), 141-149.

Kampa, M., \& Castanas, E. (2008). Human health effects of air pollution. Environmental pollution, 151(2), 362-367.

Kulkarni, M. G., \& Dalai, A. K. (2006). Waste cooking oil an economical source for biodiesel: a review. Industrial \& engineering chemistry research, 45(9), 2901-2913.

Lapinskienè, A., Martinkus, P., \& Rèbždaitè, V. (2006). Eco-toxicological studies of diesel and biodiesel fuels in aerated soil. Environmental Pollution, 142(3), 432-437.

Lin, Y. C., Lee, W. J., \& Hou, H. C. (2006). PAH emissions and energy efficiency of palmbiodiesel blends fueled on diesel generator. Atmospheric Environment, 40(21), 3930-3940.

Lin, Y. F., Wu, Y. P. G., \& Chang, C. T. (2007). Combustion characteristics of waste-oil produced biodiesel/diesel fuel blends. Fuel, 86(12), 1772-1780.

López, B. C., Cerdán, L. E., Medina, A. R., López, E. N., Valverde, L. M., Peña, E. H.,... \& Grima, E. M. (2015). Production of biodiesel from vegetable oil and microalgae by fatty acid extraction and enzymatic esterification. Journal of bioscience and bioengineering, 119(6), 706-711.

Luković, N., Knežević-Jugović, Z., \& Bezbradica, D. (2011). Biodiesel fuel production by enzymatic transesterification of oils: recent trends, challenges and future perspectives. Alternative Fuel, 1.

Mahesh, S. E., Ramanathan, A., Begum, K. M. S., \& Narayanan, A. (2015). Biodiesel production from waste cooking oil using $\mathrm{KBr}$ impregnated $\mathrm{CaO}$ as catalyst. Energy Conversion and Management, 91, 442-450.

Masjuki, H., Abdulmuin, M. Z., \& Sii, H. S. (1996). Investigations on preheated palm oil methyl esters in the diesel engine. Proceedings of the Institution of Mechanical Engineers, Part A: Journal of Power and Energy, 210(2), 131-138. 
McMichael, A. J. (2000). The urban environment and health in a world of increasing globalization: issues for developing countries. Bulletin of the World Health Organization, 78(9), 11171126.

Micklich, N., Gregorová, R., Bannikov, A. F., Baciu, D. S., Grădianu, I., \& Carnevale, G. (2016). Oligoremora rhenana ngn sp., a new echeneid fish (Percomorpha, Echeneoidei) from the Oligocene of the Grube Unterfeld ("Frauenweiler") clay pit. PalZ, 90(3), 561-592.

Mofijur, M., Masjuki, H. H., Kalam, M. A., Rasul, M. G., Atabani, A. E., Hazrat, M. A., \& Mahmudul, H. M. (2015). Effect of biodiesel-diesel blending on physico-chemical properties of biodiesel produced from Moringa oleifera. Procedia Engineering, 105, 665669.

Mofijur, M., Rasul, M. G., Hyde, J., Azad, A. K., Mamat, R., \& Bhuiya, M. M. K. (2016). Role of biofuel and their binary (diesel-biodiesel) and ternary (ethanol-biodiesel-diesel) blends on internal combustion engines emission reduction. Renewable and Sustainable Energy Reviews, 53, 265-278.

Nalgundwar, A., Paul, B., \& Sharma, S. K. (2016). Comparison of performance and emissions characteristics of DI CI engine fueled with dual biodiesel blends of palm and jatropha. Fuel, $173,172-179$.

Paredes V. (2015). Estado del desarrollo de biocumbustibles en Ecuador. Organización Latinoamericana de Energía. Olade.

Pasqualino, J. C., Montane, D., \& Salvado, J. (2006). Synergic effects of biodiesel in the biodegradability of fossil-derived fuels. Biomass and bioenergy, 30(10), 874-879.

Robalino-López, A., Mena-Nieto, A., \& García-Ramos, J. E. (2014). System dynamics modeling for renewable energy and CO 2 emissions: A case study of Ecuador. Energy for Sustainable Development, 20, 11-20.

Rocha-Hoyos, J., Tipanluisa, L. E., Reina, S. W., \& Ayabaca, C. R. (2017). Evaluación del Sistema de Tracción en un Vehículo Eléctrico Biplaza de Estructura Tubular. Información tecnológica, 28(2), 29-36.

Saba, T., Estephane, J., El Khoury, B., El Khoury, M., Khazma, M., El Zakhem, H., \& Aouad, S. (2016). Biodiesel production from refined sunflower vegetable oil over KOH/ZSM5 catalysts. Renewable Energy, 90, 301-306.

Sheehan, D. V., Lecrubier, Y., \& Sheehan, K. H. (1998). The mini-international neuropsychiatric interview (MINI): 995 the development and validation of a structured diagnostic psychiatric interview for DSM-IV and ICD-10. J Clin 996 Psychiatry. 59: 22, 33, 997.

Siegel, I., Yaghoubzadeh, E., Keskey, T. S., \& Gleicher, N. (1987). Cytotoxic effects of free fatty acids on ascites tumor cells. Journal of the National Cancer Institute, 78(2), 271-277. 
Sierra, J. C. (2016). Estimating road transport fuel consumption in Ecuador. Energy Policy, 92, 359-368.

Tandazo. (2015). Reflexiones en torno a la energía y su entorno. Petróleo\&Gas. ISSN 1390 8812 No. 004. pp 9-16.

Wang, Y., Ou, S., Liu, P., \& Zhang, Z. (2007). Preparation of biodiesel from waste cooking oil via two-step catalyzed process. Energy conversion and management, 48(1), 184-188.

Wang, Y., Ou, S., Liu, P., Xue, F., \& Tang, S. (2006). Comparison of two different processes to synthesize biodiesel by waste cooking oil. Journal of Molecular Catalysis A: Chemical, 252(1), 107-112.

World Bank. (2011). Statistics and national referents homepage. Web site http://web.worldbank.org, http://data.worldbank.org/country/ecuador.

Xiao, M. A. N., Mathew, S., \& Obbard, J. P. (2009). Biodiesel fuel production via transesterification of oils using lipase biocatalyst. Gcb Bioenergy, 1(2), 115-125.

Yacobucci, B. D., \& Schnepf, R. D. (2007, March). Ethanol and biofuels: agriculture, infrastructure, and market constraints related to expanded production. Congressional Research Service, Library of Congress.

Zhang, Y., Dube, M. A., McLean, D. D. L., \& Kates, M. (2003). Biodiesel production from waste cooking oil: 1. Process design and technological assessment. Bioresource technology, 89(1), 1-16. 